\title{
Metamagnetism in manganate magnesium ferrite
}

\author{
Jozef Sláma*, Jozef Pal'a*, Martin Šoka, Jan Lokaj ${ }^{* *}$
}

\begin{abstract}
The metamagnetic properties of the manganese magnesium ferrites having the general formula $\mathrm{Mn}_{0.7} \mathrm{Mg}_{0.3} \mathrm{Fe}_{2} \mathrm{O}_{4}$ prepared by the standard ceramic technique have been studied. It is proposed that when a change of temperature at adequate magnetic field is applied in a $\mathrm{Mn}_{0.7} \mathrm{Mgg}_{0.3} \mathrm{Fe}_{2} \mathrm{O}_{4}$ a magnetic phase transition will be generated, giving rise to an antiferromagnetic (AFM) state from ferrimagnetic (FM) phase. The critical transition field $H_{\mathrm{ac}}=300 \mathrm{~A} / \mathrm{m}$ was estimated for critical magnetization curve of transition from the metamagnetic behavior to FM behavior of sample. The FM to AFM transition in these ferrites is accompanied by a Néel type to Yafet-Kittel type transition and gradual spin ordering changes of the unit cell volume. The application of an external magnetic field to the low-temperatures AFM state causes the sample to reset to the original FM state.
\end{abstract}

K e y w or d s: metamagnetism, MnMg ferrite, magnetic cloak

\section{Introduction}

The prospect of tunable magnetic properties has inspired researchers to build novel metamagnetic materials with different characteristics. Metamagnetic materials have great potential for technological applications. The metamagnetic properties of some of the manganese magnesium ferrites are a matter of our interest for their fundamental physics and for their potential technological applications. These ferrites show different magnetic properties at different temperatures. Among spinel ferrites, $\mathrm{Mn}_{1-x} \mathrm{Mg}_{x} \mathrm{Fe}_{2} \mathrm{O}_{4}$ (MnMg ferrite) has the structure and magnetic properties that strongly depends on the ratio of $\mathrm{Mn}^{2+}$ to $\mathrm{Mg}^{2+}$ ions [1-5]. MnMg ferrite has a mixed spinel structure. Due to the special structures, $\mathrm{MnMg}$ ferrite samples with some of properties such as high permeability and saturation magnetization, low core loss have been investigated for various applications such as data storage, tissue targeting and electronic devices. One class of $\mathrm{MnMg}$ ferrites due to their change of ground states under magnetization can become also attractive in the metamagnetic systems [6]. Aiming to the application of the heating effect, we focus on studying the effect of magnetic field on the selected magnetic properties of $\mathrm{MnMg}$ ferrite.

\section{Experimental}

$\mathrm{MnMg}$ ferrites were fabricated by a conventional ceramic processing method. The mixtures of $\mathrm{Fe}_{2} \mathrm{O}_{3}, \mathrm{MnO}$ and $\mathrm{MgO}$, were sintered at $1200{ }^{\circ} \mathrm{C}$. Studied $\mathrm{Mn}_{0.7} \mathrm{Mg}_{0.3}$ $\mathrm{Fe}_{2} \mathrm{O}_{4}$ samples have rectangular hysteresis loop. Samples were of toroidal shape with inner and outer diameters 3.9 $\mathrm{mm}$ and within interval 6.15 to $6.10 \mathrm{~mm}$, respectively.
The sample was measured using SU-3500 SEM equipped with the EDX system under standard conditions. The quantity of elements present in the sample was obtained from three different spots randomly chosen. The magnetization curves were measured by a conventional experimental setup. Magnetic Barkhausen noise (MBN) and hysteresis loops measurements were made using the system described in detail in $[7,8]$. The system used a magnetising coil wound directly on the sample. Sinusoidal magnetising current of frequency $20 \mathrm{~Hz}$ was applied during the measurement. The MBN was detected by a sensing coil wound on the sample. Signal of the sensing coil was amplified and passed through an analogue band-pass filter with cut-off frequencies of $10 \mathrm{kHz}$ and $100 \mathrm{kHz}$. Additional processing by digital high-pass filter of cut-off frequency $10 \mathrm{kHz}$ was used to suppress sufficiently the interference by magnetising frequency signal. The root mean square (RMS) value and the profile of $\mathrm{MBN}$ signal were determined during the half of magnetisation cycle. These parameters of MBN were averaged over 10 cycles. Intensity of applied field was calculated from the current flowing through the magnetizing coil, while magnetic flux density was obtained from the signal of the sensing coil amplified by an analogue preamplifier. The experimental set-up allows usage of analogue (hardware) as well as digital (software) feedback to control the waveform shape of either exciting field $H(t)$ or flux density $B(t)$.

\section{Results and discussion}

Measured result could be discussed assuming the following cation distribution in the $\mathrm{Mn}_{0.7} \mathrm{Mg}_{0.3} \mathrm{Fe}_{2} \mathrm{O}_{4}$ sample: whilst 0.008 of $\mathrm{Mg}^{2+}, 0.432 \mathrm{Fe}^{3+}$ and $0.56 \mathrm{Mn}^{2+}$ ions occupy the A site, the 0.14 of $\mathrm{Mn}^{2+}, 1.568 \mathrm{Fe}^{3+}$ and

*Institute of Electrical Engineering, Faculty of Electrical Engineering and Information Technology, Slovak University of Technology in Bratislava, Slovakia, jozef.slama@stuba.sk **Institute of Material Science, Faculty of Material Science and Technology in Trnava, Slovak University of Technology in Bratislava, Slovakia, jan.lokaj@stuba.sk 


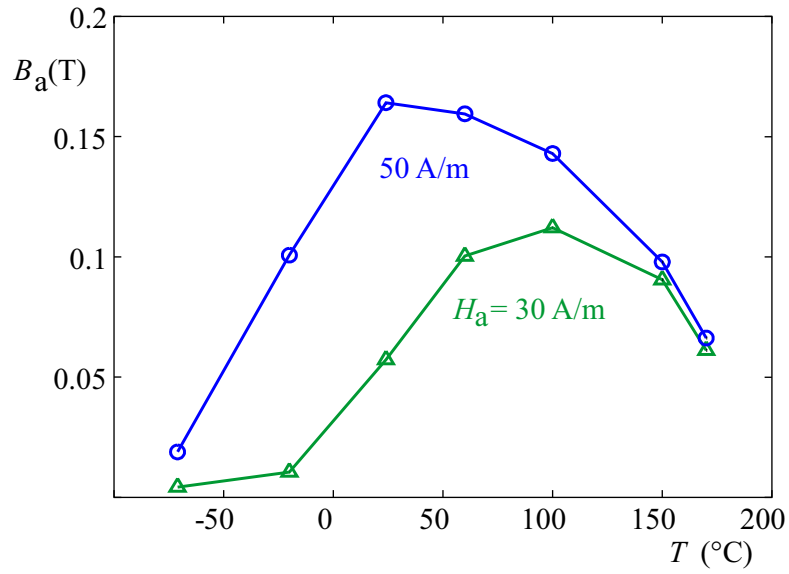

Fig. 1. The amplitude magnetic flux density $B_{\mathrm{a}}$ as functions of temperature $T$ for applied fields $H_{\mathrm{a}}=30$ and $50 \mathrm{~A} / \mathrm{m}$

$0.292 \mathrm{Mg}^{2+}$ ions occupy the B site in the spinel lattice. The total magnetic moment can be influenced by YafetKittel (Y-K) triangular magnetic ordering on B site ions leading to the change in A-O-B superexchange interactions due to changing temperature of sample at adequate applied fields. Dependences of magnetic flux density amplitude $B_{\mathrm{a}}$ on temperature $T$ of $\mathrm{MnMg}$ ferrite at various magnetic field amplitudes $H_{\mathrm{a}}$ and frequency $f=20$ $\mathrm{Hz}$ were measured. The ferrite sample was heated from the room tempeature up to Curie point $T_{\mathrm{C}}=190^{\circ} \mathrm{C}$, then cooled down to $-71^{\circ} \mathrm{C}$. Below the critical field $H_{\mathrm{ac}}=H_{\mathrm{a}}=300 \mathrm{~A} / \mathrm{m}$, the evolution of the behaviours of flux density $B_{\mathrm{a}}$ as function of $T$ is quite sensitive to field strength as well as the temperature at which the field is applied.

Both $B_{\mathrm{a}}(T)$ and magnetization $M(T)$ dependences measured below the critical field have falling values up to zero if temperature is decreasing and have metamagnetic character. From several measured $\mathrm{B}_{\mathrm{a}}(T)$ dependences the two selected curves with qualitative similar temperature behaviors are depicted in Fig. 1. These $B_{\mathrm{a}}(T)$ plots were measured in the applied field of 30 and $50 \mathrm{~A} / \mathrm{m}$, at $f=$ $20 \mathrm{~Hz}$. As example, the $B_{\mathrm{a}}(T)$ dependence exhibits the magnetic field temperature phase diagram for applied field $H_{\mathrm{a}}=30 \mathrm{~A} / \mathrm{m}$. That $B-T$ diagram is found to be composed of three different magnetic basic states. The phase diagram can be explained by a Y-K canting in $\mathrm{B}$ sites.

This opinion corresponds to finding that the magnetization results exhibit non-collinear ferrimagnetic structure for $\mathrm{Mn}_{x}$ content $x>0.3$ in $\mathrm{MnMg}$ ferrites [4]. The $B_{\mathrm{a}}(T)$ plot decreasing is marked by a rapid drop in $B_{\mathrm{a}}$ values roughly below $T=60^{\circ} \mathrm{C}$ in Fig. 1 .

A falling of $B_{\mathrm{a}}(T)$ with decreasing $T$ below $-20^{\circ} \mathrm{C}$ indicates the metamagnetic transition (MT) from cantedferrimagnetic (CFM) to the canted-antiferromagnetic (CAFM) state up to lowest temperature, even to $-273^{\circ} \mathrm{C}$ at $H_{\mathrm{a}}<30 \mathrm{~A} / \mathrm{m}$. The $T_{\mathrm{t}}=-20^{\circ} \mathrm{C}$ is a field dependent temperature transition between the two basic magnetic phases.
The data above $-20^{\circ} \mathrm{C}$ up to $100^{\circ} \mathrm{C}$ correspond probably to CFM state at $H_{\mathrm{a}}=30 \mathrm{~A} / \mathrm{m}$, because the energy required to set the antiparallel spin alignment in Néel type is higher than that required in $\mathrm{Y}-\mathrm{K}$ type order. The observed $B_{\mathrm{a}}(T)$ increase is due to saturation magnetization $M_{\mathrm{s}}$ increase, what indicates that the canting angle $(\theta)$ at $B$ site decreases as consequence of temperature increasing, yielding higher value of $M_{\mathrm{s}}$ given by

$$
M_{\mathrm{S}}(T)=M_{\mathrm{B}}(T) \cos \theta(T)-M_{\mathrm{A}}(T)
$$

and likewise yielding higher values of $B_{\mathrm{a}}(T)$. When the temperature is increased, the angle $\theta$ is decreased and moments of ions occupied in $B$-site are gradually oriented mutually parallel and simultaneously antiparallel to moments of ions that occupy $A$-sites. The observed increase in induction $B_{\mathrm{a}}(T)$ owing to $M_{\mathrm{s}}(T)$ growth, with increase in temperature implies, that spin ordering gradually changes from Y-K type to Néel type. Thus the $B_{\mathrm{a}}(T)$ plot above $T=100^{\circ} \mathrm{C}$ with colinear ferrimagnetic ground state up to $T_{\mathrm{C}}=190^{\circ} \mathrm{C}$ arises. The third basic phase above $T_{\mathrm{C}}$ is paramagnetic. In Fig.1 the second $B_{\mathrm{a}}(T)$ dependence measured at applied field $H_{\mathrm{a}}=50 \mathrm{~A} / \mathrm{m}$ exhibits the qualitatively similar magnetic field temperature phase diagram as was described above for $H_{\mathrm{a}}=30 \mathrm{~A} / \mathrm{m}$.

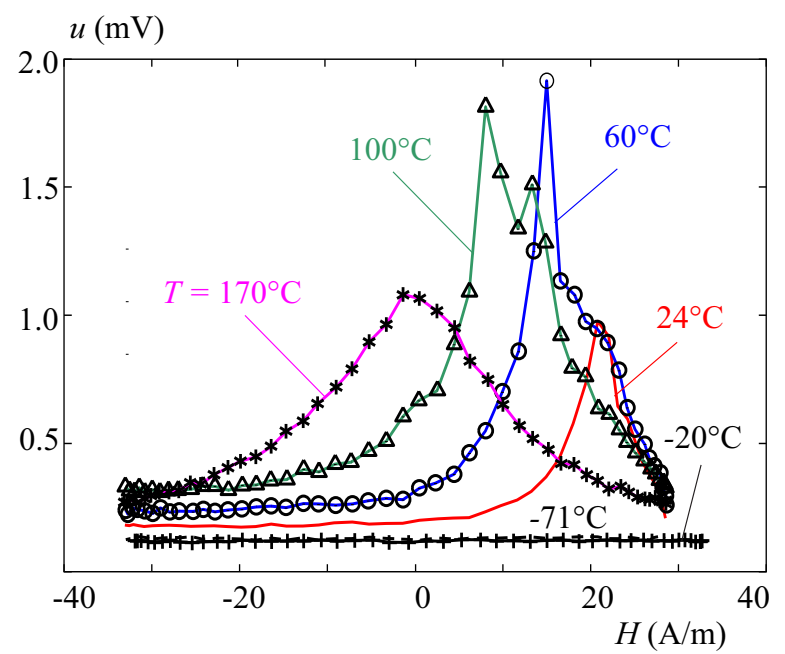

Fig. 2. Barkhausen noise profile(envelope)for $H_{\mathrm{a}}=30 \mathrm{~A} / \mathrm{m}$

MBN technique was used as a viable method for the identification of phase transition between FM and AFM ground states of the both magnetic phases of $B_{\mathrm{a}}(T)$ dependence measured at $H_{\mathrm{a}}=30 \mathrm{~A} / \mathrm{m}$ and shown in Fig. 1. From the measurement at selected different temperatures of sample (Fig. 2), the zero MBN distribution was found for $T=-20^{\circ} \mathrm{C}$ and $-71^{\circ} \mathrm{C}$. This means that there are no ferromagnetic domains as well no motions of domain walls, respectively, below $T=-20^{\circ} \mathrm{C}$. The MBN distributions were evident from $T>24^{\circ} \mathrm{C}$, and it is confirmation of FM state and domain walls motion in higher temperatures. All MBN profiles have one peak, which is located around the coercive field. The missing 


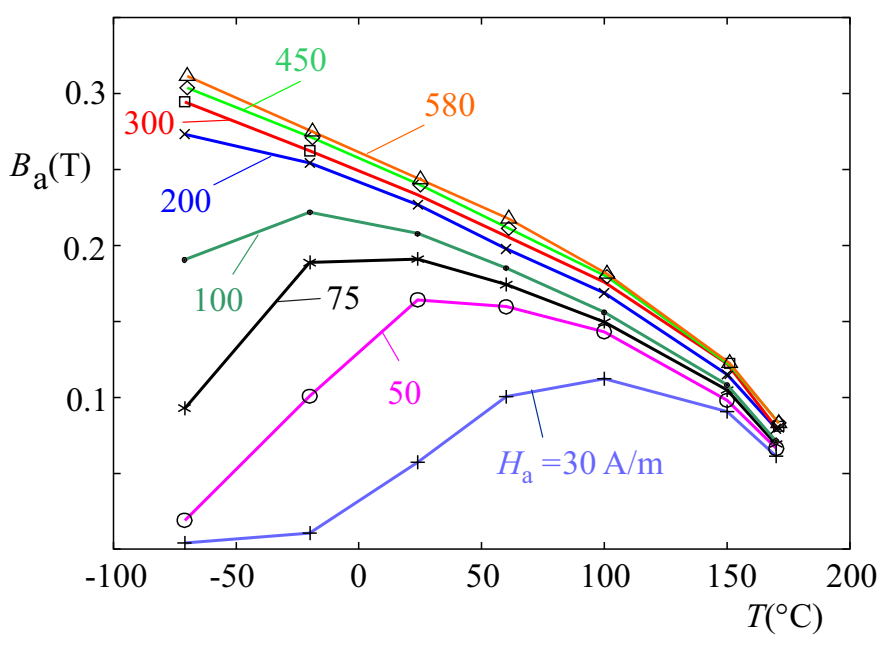

Fig. 3. The amplitude magnetic flux density $B_{\mathrm{a}}$ as functions of temperature $T$ for applied fields $H_{\mathrm{a}}=30,50,75,100,200,300,450$ and $580 \mathrm{~A} / \mathrm{m}$ at frequency $f=20 \mathrm{~Hz}$

of $B_{\mathrm{a}}(T)$ and $M(T)$ behaviors can indicate increasing of the canting angle $\theta$ in the course of falling temperature, see (1), and that the sample has CAFM ground state in all temperatures below $T=-20^{\circ} \mathrm{C}$. The $\mathrm{MBN}$ profile curves are all negatively skewed to the left at $T=24$, 60 and $100^{\circ} \mathrm{C} . \mathrm{MBN}$ profile at $T=24^{\circ} \mathrm{C}$ is shifted and skewed to highest intensity values of applied field amplitude $H_{\mathrm{a}}=30 \mathrm{~A} / \mathrm{m}$. The other MBN profiles have shifted values of profile to lower intensities of applied field amplitude for temperatures $T=60$ and $100^{\circ} \mathrm{C}$. Distribution of MBN profile depicted at $T=170^{\circ} \mathrm{C}$ is roughly a normal profile with values drifted to lowest intensities of applied field.

One can see that the skewness and shift of the MBN profile is temperature dependent and connected with the threshold field $H_{\mathrm{t}}$ at which the domain walls are activated. The $H_{\mathrm{t}}$ values are decreased with temperature and they are determined by magnetic anisotropy energy. As consequence of that, the decreasing of $H_{\mathrm{t}}$ values shows that anisotropy is also falling with temperature.

The hysteresis loops were measured at selected sequential temperatures $T=-71,24,60,100$ and $170^{\circ} \mathrm{C}$ for field $H_{\mathrm{a}}=30 \mathrm{~A} / \mathrm{m}$. Consequently these hysteresis loops were connected with $B_{\mathrm{a}}(T)$ dependence in Fig. 1, measured at $H_{\mathrm{a}}=30 \mathrm{~A} / \mathrm{m}$. The openning of loop did not arise at temperatures $T=-71,-20^{\circ} \mathrm{C}$. The opened loops were measured at higher $T$. The loop decays at $T_{\mathrm{C}}=190^{\circ} \mathrm{C}$. Figure 3 shows the temperature dependences of magnetic flux density amplitude $B_{\mathrm{a}}(T)$ for selected magnetic field amplitudes $H_{\mathrm{a}}=30,50,75,100$, $200,300,450$ and $580 \mathrm{~A} / \mathrm{m}$. By cooling the sample, $B_{\mathrm{a}}(T)$ curves at field $H_{\mathrm{a}}=300,450$ and $580 \mathrm{~A} / \mathrm{m}$ keep increasing, with temperature decreasing down to $-71^{\circ} \mathrm{C}$. $B_{\mathrm{a}}(T)$ dependences at lower amplitudes of applied field $H_{\mathrm{a}}<300 \mathrm{~A} / \mathrm{m}$ exhibit different behavior. Below the $H_{\mathrm{a}}=300 \mathrm{~A} / \mathrm{m}$, the evolution of the $B_{\mathrm{a}}$ as function of $T$ is quite sensitive to field strength as well as the temperature at which the field is applied. The $B_{\mathrm{a}}(T)$ dependences measured below the constant field $300 \mathrm{~A} / \mathrm{m}$ have metamagnetic character. From the overview, the $B_{\mathrm{a}}(T)$ curve measured at the critical constant field $H_{\mathrm{ac}}=300 \mathrm{~A} / \mathrm{m}$ presents metamagnetic transition curve, above that the $B_{\mathrm{a}}(T)$ dependences measured at field $H_{\mathrm{a}}=450$ and 580 $\mathrm{A} / \mathrm{m}$ present already collinear FM state for all temperatures.

The temperature dependences of the RMS value of MBN were measured at $H_{\mathrm{a}}=30,75,125,200,250$, 300, 450, $580 \mathrm{~A} / \mathrm{m}$ for $f=20 \mathrm{~Hz}$. Comparison of the RMS analysis of MBN with all $B_{\mathrm{a}}(T)$ curves depicted in Fig. 3 showed that the magnetization processes are prevalently governed by domain wall reversal mechanism up to external field $H_{\mathrm{a}}=580 \mathrm{~A} / \mathrm{m}$. In $\mathrm{MnMg}$ ferrite the effects of the crystalline anisotropy can be important when the applied magnetic field is small and magnetization reverses by domain walls. Therefore, metamagnetic transition from collinear ferrimagnetic state to CFM one (at $T=100^{\circ} \mathrm{C}$ ), and after that to CAFM ground state (at $T_{\mathrm{t}}=-20^{\circ} \mathrm{C}$ in Fig. 1) may be related to the temperature dependence of the anisotropy field that increases on lowering the temperature, for applied field $H_{\mathrm{a}}=30 \mathrm{~A} / \mathrm{m}$. Below $-20^{\circ} \mathrm{C}$, this metamagnet displays the zero response induction and magnetization in the applied fields $H_{\mathrm{a}} \leq 30 \mathrm{~A} / \mathrm{m}$, what means practical demonstration of a magnetic version of an invisible magnetic cloak.

\section{Conclusion}

Measured $B_{\mathrm{a}}(T)$ curves show a ferrimagnetic relation at applied field $H_{\mathrm{a}} \geq 300 \mathrm{~A} / \mathrm{m}$, in temperature range from $-71^{\circ}$ up to $T_{\mathrm{C}}=190^{\circ} \mathrm{C}$. The temperature dependences of magnetic induction $B_{\mathrm{a}}(T)$ of the measured samples show a metamagnetic transitions with three regions below critical applied field $H_{\mathrm{ac}}=300 \mathrm{~A} / \mathrm{m}$ in soft magnetic $\mathrm{Mn}_{0.7} \mathrm{Mg}_{0.3} \mathrm{Fe}_{2} \mathrm{O}_{4}$ ferrite. The first low temperature region belongs to the antiferromagnetic phase, the second region to ferrimagnetic phase and third region is paramagnetic state above Curie temperature. The transitions from CFM state to CAFM state on the thermomagnetic $B_{\mathrm{a}}(T)$ curves can be explained with the help of an approach to the Yafet-Kittel triangular magnetic ordering on the $B$-site ions leading to the change in exchange interactions due to changing temperature of sample at adequate applied fields. The MBN method was used to qualitative study of the metamagnetic transitions of the measured $\mathrm{MnMg}$ ferrite. FM phase arising out of change from the AFM state was identified by the intensity of MBN.

\section{Acknowledgements}

This work was supported in part by the VEGA agency of the Slovak Republic (projects 1/0405/16, 1/0122/16 and $1 / 0571 / 15$ ), and partly by the Slovak Research and Development Agency (contracts APVV-15-0257, APVV0062-11 and APVV-16-0059). 


\section{REFERENCES}

[1] M.-F. Kuo, Y.-H. Hung and J.-Y. Huang, "Substitution effects on magnetic properties of $\mathrm{Mg}_{1.3-x} \mathrm{Mn}_{x} \mathrm{Al}_{y} \mathrm{Fe}_{1.8-y} \mathrm{O}_{4}$ ", AIP Advances, vol. 7, 056104-1-6, (2017).

[2] O. M. Hemeda, N. Y. Mostafa, O. H. Abd Elkader and M. A. Ahmed, "Solubility limits MnMg ferrites system under hydrothermal conditions", Journal of Magnetism and Magnetic Materials, vol. 364, pp. 3946, (2014).

[3] N. Lwin, A. Fauzi, M. N. S. Sreekantan and R. Othman, "Physical and electromagnetic properties of nanosized Gd substituted MgMn ferrites by solution combustion method", Physica B: Condensed Matter, vol. 461, pp. 134139, (2015).

[4] A. A. Pandit, A. R. Shitre, D. R. Shengule and K. M. Jadhav, "Magnetic and dielectric properties of $\mathrm{Mg}_{1+x} \mathrm{Mn}_{x}, \mathrm{Fe}_{2-2 x}, \mathrm{O}_{4}$ ferrite system", Journal of Materials Science, vol. 40, pp 423428, (2005).

[5] G. Kumar, V. P. Singh, A. Kumar, J. Shah, S. Kumar, B. S. Chauhan, R. K. Kotnala and M. Singh, "Estimation of magnetic interaction substituted $\mathrm{Mg}-\mathrm{Mn}$ ferrites syntetized via citrate precursor technique", Advanced Materials Letters, vol. 6, pp. 828-833, (2015).

[6] J. Sláma, M. Šoka and V. Jančárik, "Metamagnetism MnMg Ferrite: Medium for Magnetic Cloak and Shielding", Journal of Electrical Engineering, vol. 66, pp. 120-12, (2015).

[7] J. Pal'a and J. Bydžovský, "An alternative method to remove excitation field interference from magnetic Barkhausen noise", Journal of Magnetism and Magnetic Materials, vol. 361, pp. 8893, (2014).

[8] A. J. Moses, H. V. Patel and P. I. Williams, "AC Barkhausen Noise Electrical Steels: Influence of Sensing Technique on Interpretation of Measurements", Journal of Electrical Engineering, vol. 57, pp. 3-8, (2006).

Received 13 February 2018

Jozef Sláma (prof, Ing, PhD), graduated from Faculty of Electrical Engineering, Slovak Technical University,
Bratislava, in Solid-state Physics branch, 1963 and received the $\mathrm{PhD}$ degree in Theory of Electromagnetism in 1969. At present, he is a Professor at the Department of Electromagnetic Theory, Faculty of Electrical Engineering and Information Technology. His research activities are mainly applied magnetism, research of magnetization processes, losses and ferrites.

Jozef Pal'a was born in Martin, Slovakia, in 1976. He graduated from the Faculty of Electrical Engineering and Information Technology, Slovak University of Technology in Bratislava, in Electronics branch in 2000, and received the $\mathrm{PhD}$ degree in Theory of Electromagnetism in 2007. At present, he is with the Institute of Electrical Engineering. His areas of research include magnetic measurements, Barkhausen noise analysis and computer modelling of magnetic fields.

Martin Šoka (Ing, PhD), born in Bratislava, Slovakia, in 1982, graduated from the Faculty of Electrical Engineering, Slovak University of Technology in Bratislava, from microelectronics branch, in 2007, and received the $\mathrm{PhD}$ degree in Theory of Electromagnetism, in 2011. Since 2007 he has been with the Department of Electromagnetic Theory at the Institute of Electrical Engineering. He teaches electric circuits. In research, he is involved in measurement of the temperature dependences of magnetic susceptibility.

Jan Lokaj (Prof, Ing, PhD,) born in Slovakia 1951. Graduated at the Faculty of Electrical Engineering in 1974 and received the $\mathrm{PhD}$ degree in 1982 in the field of Crystal structure analysis. At present he teaches Applied physics at the Faculty of Material science and Technology. His research is focused on the SEM and Microanalysis of progressive ceramics materials, reparation and analysis of bimetals welded by explosion as well as the crystal structure analysis of novel organic compounds. 